\title{
Optimal management of hepatorenal syndrome in patients with cirrhosis
}

This article was published in the following Dove Press journal:

Hepatic Medicine: Evidence and Research

19 June 2010

Number of times this article has been viewed

\author{
Paolo Angeli \\ Filippo Morando \\ Department of Clinical and \\ Experimental Medicine, University \\ of Padova, Italy
}

Correspondence: Paolo Angeli

Department of Clinical and Experimental Medicine, University of Padova,

Via Giustiniani 2, cap. 35I00 Padova, Italy Email pangeli@unipd.it

\begin{abstract}
Hepatorenal syndrome (HRS) is a functional renal failure that often occurs in patients with cirrhosis and ascites. HRS develops as a consequence of a severe reduction of effective circulating volume due to both an extreme splanchnic arterial vasodilatation and a reduction of cardiac output. There are 2 different types of HRS. Type 1 HRS, which is often precipitated by a bacterial infection, especially spontaneous bacterial peritonitis, is characterized by a rapidly progressive impairment of renal function. Despite its functional origin, the prognosis of type 1 HRS is very poor. Type 2 HRS is characterized by a stable or slowly progressive renal failure so that its main clinical consequence is not acute renal failure but refractory ascites and its impact on prognosis is less negative. New treatments (vasoconstrictors plus albumin, transjugular portosystemic shunt, and molecular adsorbent recirculating system), which were introduced in the past 10 years, are effective in improving renal function in patients with HRS. Among these treatments vasoconstrictors plus albumin can also improve survival in patients with type $1 \mathrm{HRS}$. Thus, this therapeutic approach has changed the management of this severe complication in patients with advanced cirrhosis.
\end{abstract}

Keywords: cirrhosis, ascites, bacterial infections, spontaneous bacterial peritonitis, renal failure, acute renal injury, chronic kidney disease, vasoconstrictors, terlipressin, midodrine, albumin, transjugular portosystemic shunt, renal replacement therapy

\section{Introduction}

Hepatorenal syndrome (HRS) is a functional renal failure that frequently occurs in patients with cirrhosis and ascites. The prevalence of HRS in patients affected by cirrhosis and ascites was $18 \%$ after 1 year which increase to $39 \%$ at 5 years. ${ }^{1}$ In almost half the cases of HRS, one or more precipitating factors may be identified, among which there are bacterial infections (57\%), gastrointestinal hemorrhage (36\%), and therapeutic paracentesis $(7 \%)$. There is considerable evidence that HRS is related to disturbances in circulatory function, namely low values of arterial pressure which is primarily due to a peripheral arterial vasodilation mainly in the splanchnic circulation. The cause of arterial vasodilatation is an increased release of endogenous vasodilator factors triggered by portal hypertension. ${ }^{2}$ Until 1999, the prognosis for cirrhotic patients developing HRS was very poor with mortality running at around $100 \%$ in cases, and a median survival rate from the moment of onset of 2 weeks. ${ }^{1}$ Thereafter, some new effective treatments of HRS which improve survival have been introduced with promising results. 


\section{Diagnosis of HRS}

HRS is characterized by (1) marked renal vasoconstriction with a consequent reduction in renal plasma flow and glomerular filtration rate (GFR), (2) the absence of histological changes other than a reflux of proximal convoluted epithelium into Bowman's space, ${ }^{3}$ and (3) preserved tubular renal function. Due to its functional nature, HRS has no specific hallmarks. The diagnosis of HRS is, therefore, made by excluding the occurrence of the other forms of renal failure. The first diagnostic criteria for HRS proposed by the "International Ascites Club"4 have recently been reexamined ${ }^{5}$ (Figure 1). The main changes in the new diagnostic criteria of $\mathrm{HRS}^{5}$ are (1) the removal of minor diagnostic criteria, such as serum sodium concentration, urinary sodium excretion, the ratio of urinary to plasma osmolality, which are no longer considered essential for diagnosis of HRS and (2) the removal of ongoing bacterial infection as an exclusion criteria for the diagnosis of HRS. Other important changes are (1) plasma volume expansion should be no longer performed with saline solution but with albumin and (2) creatinine clearance is no longer to be considered as a tool for the diagnosis since it offers little advantages in terms of accuracy in the estimation of renal function as compared with the use of serum creatinine which, in turn, is quite simpler in clinical practice. Likewise, the creatinine-based Cockcroft and MDRD equations, which are widely used in the general population to estimate GFR, were found to be inaccurate in cirrhotic patients and were not considered among the diagnostic criteria of HRS. ${ }^{5}$ The presence of shock, even of septic shock, as well as previous treatment with nonsteroidal anti-inflammatory drugs (NSAIDs), other nephrotoxic drugs (ie, aminoglycosides) and vasodilators (ie, nitrates, prazosin, and renin-angiotensin system inhibitors) are still considered exclusion criteria for the diagnosis of HRS as well as the presence of proteinuria, hematuria or ultrasound alterations of the kidneys. ${ }^{4,5}$ Recently, the Acute Kidney Injury Network (AKIN) has proposed the

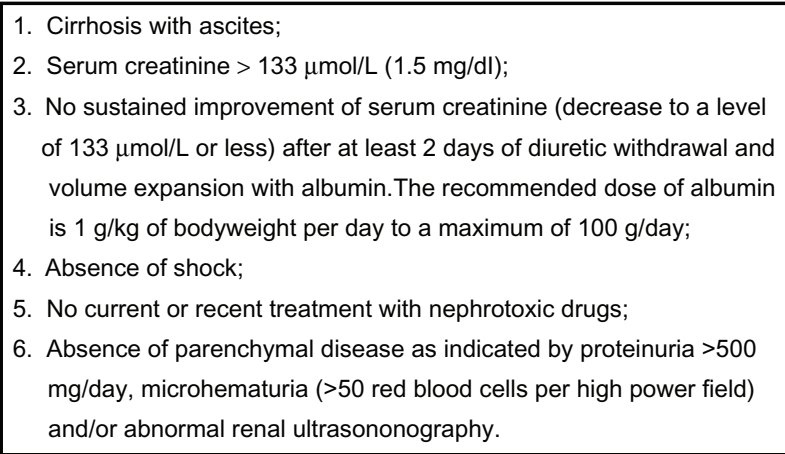

Figure I New diagnostic criteria of hepatorenal syndrome. term "acute kidney injury" (AKI) and a set of consensus criteria to define acute renal failure, taking into account recent evidence that smaller increments in serum creatinine have impact on prognosis. ${ }^{6,7} \mathrm{~A}$ recent review of AKI in cirrhosis has included these new terms ${ }^{8}$ but the new diagnostic criteria have not yet been applied in patients with cirrhosis. To this day, most studies and guidelines have defined renal failure on cirrhosis as a serum creatinine concentration $>1.5 \mathrm{mg} / \mathrm{dL}$ $(133 \mu \mathrm{mol} / \mathrm{L})$. However, considering that serum creatinine underestimates GFR in patients with cirrhosis, ${ }^{9}$ this definition identifies only severe renal failure without recognizing early phase on this clinical complication thereby having a potential negative impact on treatment.

Two types of HRS have been distinguished in Figure 2. Type 1 HRS is characterized by a doubling of the serum creatinine level with a final value $>2.5 \mathrm{mg} / \mathrm{dL}$ in less than 2 weeks so that its main clinical feature is acute renal failure. By contrast, in patients with type 2 HRS, which probably represents the most common form of chronic kidney disease (CKD) in cirrhosis, the degree of the impairment of renal failure is more stable over weeks or months. The presence of a refractory ascites is the main clinical problem in these patients (Figure 2). ${ }^{3,4}$ In addition, the 2 types of HRS differ substantially in prognostic terms. In fact, before the development of the new therapeutic options, the median survival of type 1 HRS was about 2 weeks. To this date, it has been slightly increased by the introduction of the use of vasoconstrictors and albumin; nevertheless, median survival of type 1 HRS still remains shorter than that of type 2 HRS which is generally around 4-6 months. ${ }^{10}$ Differences at a pathophysiological level between type 1 HRS and type 2 HRS are not fully explained. However, on clinical ground, it may be observed that type 1 HRS is often induced by the occurrence of a precipitating event, in particular by the development of a spontaneous bacterial peritonitis (SBP). ${ }^{11,12}$ Almost one-third of patients with SBP develop a nontransient form of renal failure ${ }^{11,12}$ which in most cases fully satisfy the diagnostic criteria of type 1 HRS. ${ }^{12}$ More recently, it has been observed that an AKI can precipitated in cirrhotic patients with ascites by all types of bacterial infections. In most cases, renal failure is transient and reverses with the resolution of the infection by means of antibiotic treatment. However, an AKI with the hallmarks of type 1 HRS can be precipitated not only by SBP but also by urinary tract infection and infections of the biliary or intestinal tract. ${ }^{13}$ The independent predictive factor for the development of renal failure as a consequent of bacterial infections are (1) the severity of infection, (2) the model for end stage liver disease (MELD) score at 
Type 1 HRS: rapidly progressive reduction of renal function as defined by a doubling of the initial serum creatinine to a level > $226 \mu \mathrm{mol} / \mathrm{L}$ or $2.5 \mathrm{mg} / \mathrm{dL}$ in less than 2 weeks. It may occurs spontaneously, but it can also follow a precipitating event.

Clinical pattern: acute renal failure

Type 2: is characterized by moderate renal failure (serum creatinine from 133 to $226 \mu \mathrm{mo} 1 / \mathrm{L}$ or 1.5 to $2.5 \mathrm{mg} / \mathrm{dL}$ ) with a steady or slowly progressive course.

Clinical pattern: refractory ascites

Figure 2 Clinical types of hepatorenal syndrome.

the diagnosis of infection, and (3) the lack of resolution of infection by mean of antimicrobial therapy. ${ }^{13,14}$ The risk of developing a type $1 \mathrm{HRS}$ as a result of bacterial infection is higher in patients with cirrhosis who already are suffering from type 2 HRS. ${ }^{11,12}$

\section{Pathophysiology of HRS Systemic and renal hemodynamics}

Renal arterial vasoconstriction, which is the pathophysiological basis of HRS, ${ }^{15}$ develops in the context of a marked reduction of effective circulating volume related to peripheral arterial vasodilatation. ${ }^{2}$ Splanchnic arterial vasodilatation is thought to be mainly the consequence of an increased release of endogenous vasodilators due to portal hypertension and/or hepatic failure. Among the endogenous vasodilators, nitric oxide, ${ }^{16}$ carbon monoxide,,${ }^{17}$ glucagon,,${ }^{18}$ prostacyclin, ${ }^{19}$ adrenomedullin, ${ }^{20}$ and endogenous oppiate ${ }^{21}$ seem to be the most involved. A detailed analysis of all the possible vasodilators which are involved in the pathogenesis of splanchnic arterial vasodilations goes beyond the purposes of this paper. Nonetheless, it can be hypothesized that several endogenous vasodilators are able to contribute to splanchnic arterial vasodilatation throughout the course of cirrhosis and the relative role of each of them can vary in the different stages of the liver disease. ${ }^{22}$ In advanced cirrhosis, systemic arterial pressure is maintained despite the splanchnic arterial vasodilatation through the activation of the systemic vasoconstrictor systems, including the renin-angiotensin system, the sympathetic nervous system and, later, the nonosmotic release of vasopressin. ${ }^{2}$ These compensatory mechanisms contribute to maintain a relatively normal systemic arterial pressure but are thought to be responsible for functional renal abnormalities such as renal sodium retention, renal water retention and, finally, renal arterial vasoconstriction which is the pathophysiological basis of HRS. ${ }^{2}$

The involvement of endogenous vasoconstrictor systems induced by the reduction of effective circulating volume in the development of HRS is documented both at clinical ${ }^{23-25}$ and experimental level. ${ }^{26}$ The most recent advances in the pathogenesis of HRS have focused mainly on the finding that in patients with cirrhosis and ascites who are developing a type $1 \mathrm{HRS}$, a cardiac output is relatively insufficient to prevent the severe reduction of effective circulating volume due to the splanchnic arterial vasodilatation. In the early stages of the liver disease, the decrease in systemic vascular resistance due to arterial splanchnic vasodilatation is compensated by the increase in cardiac rate and in cardiac output which define a hyperdynamic circulation. But, as the liver disease progresses with a further increase of portal hypertension and further impairment of hepatic function, the cardiac output becomes no longer adequate to correct the severity of the reduction of the effective blood volume due to splanchnic arterial vasodilatation (Figure 3), thereby leading to a maximal over-activation of the systemic endogenous vasoconstrictor systems. ${ }^{27,28}$ This is the pathophysiological scenario in which HRS develops. On confirming this phenomenon, additional increases in cardiac output were shown to be inadequate to compensate the reduction of effective circulating volume in patients with advanced cirrhosis. ${ }^{29}$ Moreover, cardiac output has been shown to be normal or even reduced in patients with cirrhosis and HRS or in patients with cirrhosis and refractory ascites. ${ }^{30-32}$ Systemic hemodynamics has been investigated more in detail in patients who developed type 1 HRS either as a consequence of SBP or spontaneously. The development of type 1 HRS was associated with a decrease of arterial pressure, a marked decrease of cardiac output, and a marked activation of the systemic vasoconstrictor systems, but was not associated with a further decrease of the peripheral vascular resistance. ${ }^{33}$ More recently, it has been observed that a low cardiac output is associated with a higher probability of developing a type 1 HRS and with a poor outcome. ${ }^{34}$

The above findings support the hypothesis that a hyperdynamic circulation is essential for the maintenance of an effective blood volume in patients with cirrhosis and that a decrease of cardiac output, due or not to a precipitating event such as a bacterial infection, can lead to a severe effective hypovolemia, a severe intrarenal arterial vasoconstriction and, therefore, to HRS. The reasons why cardiac output can decrease in the end stage liver disease is still unknown, but, in recent years several specific cardiac abnormalities such as reduced systolic and diastolic responses to stress stimuli, electrophysiological changes and enlargement of cardiac chambers have been recognized as the so-called "cirrhotic cardiomiopathy." ${ }^{15}$ In addition, other factors such as release of endotoxins and further release of biologically active substances such as inflammatory cytokines, nitric oxide, car- 


\section{Progressive increase in cardiac output No further increase/fall in cardiac output}

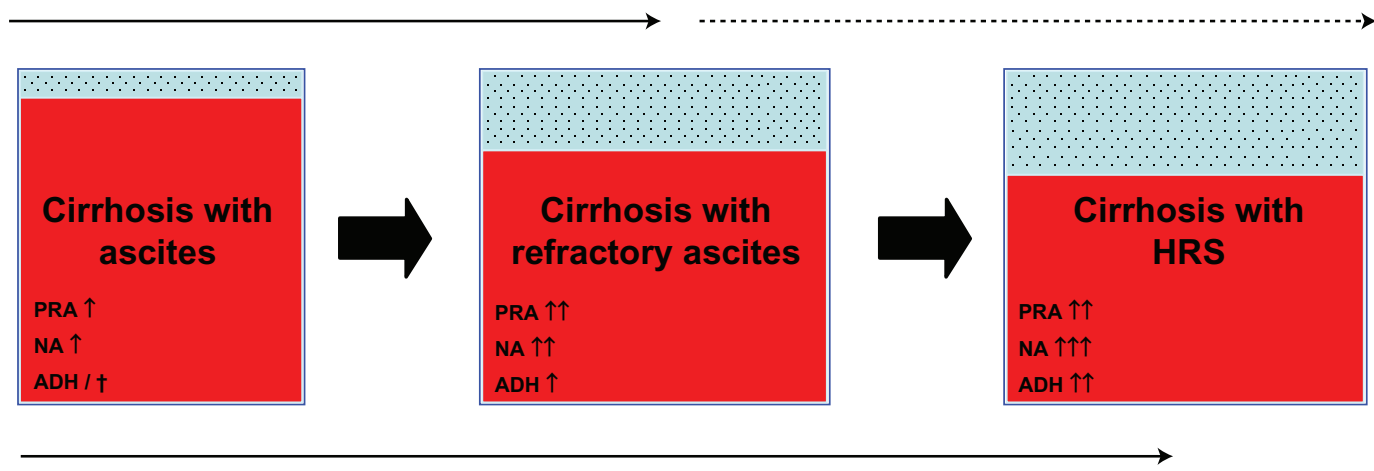

Progressive splanchnic arterial vasodilatation

Volume of the arterial vascular tree

Blood volume

\section{Reduction of effective circulating volume}

Figure 3 Pathophysiology of progressive impairment of the effective circulating volume in patients with advanced cirrhosis. Abbreviations: PRA, plasma renin activity, NA, plasma level of noradrenaline, ADH, plasma level of antidiuretic hormone.

bon monoxide, and others resulting from bacterial infection may further impair cardiac function in patients with end stage liver disease. A severe sepsis or a septic shock is often also associated with adrenal insufficiency in patients with cirrhosis and ascites and it correlates with hemodynamic instability and the development of HRS. ${ }^{36,37}$ However, several findings suggest that the reduction of cardiac output in patients with cirrhosis who are going to develop HRS is mainly related to a reduction of the venous return to the heart. First, the reduction of cardiac output in these patients is not associated with an increase in cardiopulmonary pressure. ${ }^{33,34}$ Second, the hemodynamic scenario which represents the basis of HRS may be reverted by the insertion of a transjugular intravenous (IV) portosystemic shunt (TIPS) which increases the venous return and, consequently, the cardiac output. But as far as the effect of TIPS on renal perfusion is concerned, it should be observed that reduction of portal pressure which can be obtained by TIPS insertion can improve renal perfusion directly through what has been referred "hepatorenal reflex." 38

\section{Renal vasoconstrictors and vasodilators}

The impairment of systemic hemodynamics is thought to be essential in the pathogenesis of HRS. Nonetheless, the role of intrarenal vasoconstrictors and vasodilators in the pathogenesis of HRS should not be underestimated. As far as vasoconstrictors are concerned, it has been shown that an antagonist of the endothelin's receptors produced improved renal perfusion in patients with HRS and no variation in arterial pressure systems, testifying to the fact that endothelin may act mainly as an intrarenal vasoconstrictor. ${ }^{39}$ These results have been not confirmed recently since it has been shown that the administration of nonselective endothelin receptor antagonist cause a deterioration of renal function in patients with type 2 HRS. ${ }^{40}$ Therefore, the role of endothelin in the pathogenesis of HRS needs further investigation. Other studies have reported an increased release at a strictly intrarenal level of other very potent vasoconstrictors in patients with cirrhosis and ascites, such as 20-hydroxyeicosatetraenoic acid and leukotrienes. ${ }^{41-44}$ As far as vasodilators are concerned, it should be observed that on experimental level, a renal failure may be provoked in cirrhotic animals with ascites via the administration of inhibitor releasing substances that at a renal level perform a vasodilatory action and which include prostaglandins E2 and I2 (PGE2 and PGI2), ${ }^{45}$ endogenous natriuretic peptides, ${ }^{46}$ and nitric oxide. ${ }^{45}$ Finally, it should be underlined that in cirrhotic patients with ascites, the administration of NSAIDs may cause an acute form of renal failure which is quite similar to HRS. ${ }^{47}$

\section{Treatment of patients with cirrhosis and HRS}

\section{Liver transplantation}

Orthotopic liver transplantation (OLT) represents the therapeutic option of choice in cirrhotic patients with HRS because it is able to remove its main causes which are portal hypertension and liver failure. However, the presence of HRS at the moment of the transplant compromises the transplant's 
success in terms of survival, costs, and quality of life. ${ }^{48}$ The survival rate is only slightly reduced as compared with that observed after OLT in patients without HRS $(60 \%$ vs $70 \%-80 \%$ at 3 years). Patients with HRS who undergo OLT have more complications after OLT, spend more days in intensive care unit, and have a higher in-hospital mortality. As far as renal function is concerned, it is known that, soon after OLT, GFR further decreases in patients with HRS and many of them require hemodialysis (HD; $35 \%$ as compared with $5 \%$ of patients without HRS at the time of OLT). ${ }^{48}$ Despite the disappearance of the hemodynamic and neurohumoral abnormalities by 1 month after OLT ${ }^{49}$ GFR improves only partially reaching $30-40 \mathrm{~mL} / \mathrm{min}$ by $1-2$ months. This moderate renal failure persists during the follow-up and can evolve to an end stage renal failure if the immunosuppressive strategy is not targeted towards sparing renal function (ie, avoiding the use or reducing the dose of calcineurin inhibitors). ${ }^{50}$ Nonetheless, up to the end of the 1990 s, very few patients with cirrhosis and HRS underwent OLT. Due to the rapid evolution of type $1 \mathrm{HRS}$ and its dramatic effect on survival, most patients died before OLT. The introduction of MELD has partially solved this problem since patients with high serum creatinine values have now a great priority for OLT. Nevertheless, other therapeutic options were developed either as "treatment bridge" towards OLT or as treatment in patients who are not candidates for OLT. Among them, the administration of vasoconstrictors plus albumin is the most commonly used in patients with HRS since it was proven to be an effective in the treatment of HRS in patients with cirrhosis and ascites. It has been shown that this therapeutic option increases the number of patients with type 1 HRS reaching OLT, and, after OLT, reduces the need for renal replacement therapy (RRT), improving their survival. ${ }^{51}$ This new therapeutic option as well as some others will be discussed later separately for type 1 and type 2 HRS.

Simultaneous liver-kidney (SLK) transplantation is increasing in frequency because of the concern over the negative impact of post-OLT renal failure and overall post-OLT need of RRT on survival. Since pre-OLT renal dysfunction is the main predictor of post-OLT renal failure, all candidates for OLT with renal dysfunction can been considered as potential candidates for SLK. The key is to select among them those with a renal dysfunction which will not recover after OLT. The transplant community tried to answer to this important point through 2 consensus conferences which have determined that SLK should be offered to (1) OLT candidates with end stage renal disease or with AKI for any cause including HRS who have been in RRT for more than 6-8 weeks,
(2) OLT candidates with a measured GFR $<30 \mathrm{~L} / \mathrm{min}$ for more than 3 months especially in the presence of other evidence supporting the diagnosis of CKD, and (3) patients with CKD with kidney biopsy showing fixed renal damage ( $>30 \%$ glomerulosclerosis or $30 \%$ fibrosis)..$^{52,53}$ Nonetheless, there are some evidences which suggest that the best timing for liver transplantation alone in patients with type 1 HRS to assure a post-OLT recovery of renal function is within 4 weeks from the occurrence of HRS regardless the need and the duration of RRT before OLT. ${ }^{54,55}$

\section{Other therapeutic options for type I HRS}

The administration of vasoconstrictors and albumin in patients with type 1 HRS is based on the more recent acquisitions relating to the pathophysiology of HRS. In small pilot perspective studies as well as in retrospective studies, it has been demonstrated that the prolonged use of a vasoconstrictor derived from vasopressin, ornipressin ${ }^{56,57}$ or terlipressin ${ }^{58-67}$ or of an $\alpha$-agonist vasoconstrictor (noradrenaline alone, midodrine plus octreotide) ${ }^{68-71}$ in association with human albumin is capable of recovering renal function in patients with type 1HRS (Figure 4).

Overall these studies have demonstrated that (1) a recovery of renal function can be obtained in $40 \%-60 \%$ of patients by using a vasoconstrictor plus albumin for a mean period of 10-15 days, (2) recurrence of HRS after treatment withdrawal (a sharp increase in serum creatinine within few days) occurs in approximately $20 \%$ of patients, but retreatment is often effective, and (3) in most cases dilutional hyponatremia associated with HRS improves with vasoconstrictors and albumin. Among vasopressin derivatives, to this day, terlipressin is the most widely used vasoconstrictor in the treatment of type 1 HRS. ${ }^{10,58-67}$ No experience using vasopressin exists as yet, whereas ornipressin is no longer used in patients with type 1 HRS due to its poor safety. ${ }^{56}$ Terlipressin has been used in more than 200 patients either as IV bolus moving from an initial dose of $0.5 \mathrm{mg}$ for every 4-6 hours, or continuous IV

- Be sure of the diagncsis by excluding the other forms of renal failure

- Look carefully for a precipitating event especially bacterial infections

- Remember that the clinical picture is an acute renal failure and, thus adopt all the general measures for this clinical condition

- Start treatment with vasoconstrictors plus albumin monitoring closely its efficacy and safety

- Set up high priority for liver transplant in suitable patients

- Take into account TIPS in patients without severe liver failure who failed to respond to vasoconstrictors and albumin

- Take into account techniques of renal replacement when indicated

Figure 4 Key points in the management of type I hepatorenal syndrome. 
infusion moving from an initial dose of $2 \mathrm{mg}$ twice daily. In patients without response (no significant reduction of serum creatinine within 3 days), the initial dose of terlipressin was doubled. The maximal doses of terlipressin used in the treatment of type 1 HRS were $2 \mathrm{mg}$ for every 4-6 hours by IV boluses, or $12 \mathrm{mg}$ twice daily by continuous IV infusion. Complete reversal (defined by a decrease of serum creatinine with a final value $<1.5 \mathrm{mg} / \mathrm{dL}$ ) or partial reversal (defined by a decrease of serum creatinine $>50 \%$ with a final value $\geq 1.5 \mathrm{mg} / \mathrm{dL}$ ) of type $1 \mathrm{HRS}$ was observed in almost $59 \%$ of patients. ${ }^{9}$ In 2 studies in which terlipressin was given alone, ${ }^{64,66}$ reversal of renal failure was lower than in the studies in which terlipressin was associated with albumin. So far, midodrine and octreotide have been used in 4 studies in a total of 154 patients. ${ }^{68-71}$ A complete reversal of renal failure was observed in $49 \%$ of patients. In most patients, midodrine administration started at 5-10 $\mathrm{mg}$ three times a day (tid) orally, with the aim of increasing the dose to 12.5 or $15 \mathrm{mg}$ tid if an increase of mean arterial pressure $\geq 5 \mathrm{~mm}$ $\mathrm{Hg}$ coupled with a reduction of serum creatinine was not observed. Octreotide administration started at $100 \mu \mathrm{g}$ subcutaneously tid with the aim of increasing the dose to $200 \mu \mathrm{g}$ subcutaneously tid if no response ensued. Experience of the use of IV norepinephrine in the treatment of patients with type 1 HRS is more limited but promising. ${ }^{72,73}$ Albumin should be used by starting with a priming dose of $1 \mathrm{~g} / \mathrm{kg}$ of body weight followed by $20-40 \mathrm{~g}$ /day. For an optimization of the dose of albumin it is, however, advisable to monitor central venous pressure. The decrease in serum creatinine as a result of the administration of vasoconstrictors and albumin takes several days. Therefore, the length of treatment is usually 10-15 days. Despite the normalization of serum creatinine, GFR, when specifically measured, ${ }^{68}$ remains below the normal values in most responders to treatment. Urinary sodium and free water excretion may improve in some, but not in all responders. It is noteworthy to underline that urine analysis as well as proteinuria should be monitored in nonresponders since type 1 HRS can evolve towards acute tubular necrosis (ATN). ${ }^{74}$

Finally, it should be stressed that vasoconstrictors can induce side effects in up to $40 \%$ of patients, namely diarrhea, transient abdominal pain, myocardial infarction, arrhythmia, intestinal ischemia, and circulatory overload. Severe side effects require the discontinuation of treatment in up to $10 \%$ of patients. With regards to 30 -day transplant-free survival, it varies from $43 \%$ in those treated with terlipressin and albumin ${ }^{10,58-67}$ to $48 \%-70 \%$ in those treated with midodrine, octreotide, and albumin. ${ }^{68,70,71}$ Since 30-day survival was within 3\%-33\% in untreated patients, ${ }^{70,71}$ it seems that vasoconstrictors and albumin can improve short-term survival in patients with type 1 HRS. Nevertheless, the results of the first 2 randomized controlled clinical trials (RCTs) comparing terlipressin and albumin with albumin alone do not confirm a beneficial effect of terlipressin and albumin on 1-, 2-, and 3-month survival in patients with type 1 HRS. ${ }^{75,76}$ A systematic review of all RCTs has recently confirm that vasoconstrictor drugs, in particular, terlipressin plus albumin may prolong only 15-day survival in patients with type 1 HRS. ${ }^{77}$ This result highlights the meaning of the use of vasoconstrictors and albumin as a bridge treatment towards OLT. Nonetheless, it appears more and more evident that in clinical practice, this pharmacological approach to type $1 \mathrm{HRS}$ is often used also in patients who are not candidate to OLT. ${ }^{78}$

Although interpreting the small effect of terlipressin and albumin on survival in patients with type $1 \mathrm{HRS}$, several points should be underlined. First of all, both the controlled clinical trials on the treatments of type 1 HRS have some limits. In the US study, albumin was not used on the basis of a regular schedule in both arms and there is a strong difference in the outcomes among the different participating centers. ${ }^{75}$ In the Spanish study, the size was smaller than that planned at the beginning of the study. ${ }^{76}$ Then, it should be taken into account that the prognosis in these patients is not only related to a recovery of renal function, but also to the degree of liver failure. It should be also emphasized that a marked impairment of liver function does not only represent a poor predictor for the response to treatment with vasoconstrictor and albumin ${ }^{66,67}$ but also a poor predictor for survival in patients with type 1 HRS undergoing the treatment. A score according to Child-Pugh results $>11$ implies a poor survival in spite of any eventual recovery of renal function. ${ }^{64,66,67}$ Examining this more closely, it has been recently shown that a serum total bilirubin $\geq 10 \mathrm{mg} / \mathrm{dL}$ is also a predictor of nonresponse as is as an increase in arterial pressure $<5 \mathrm{~mm} \mathrm{Hg}$ at day 3 of treatment. ${ }^{79}$

The efficacy of TIPS in the treatment of type 1 HRS has been evaluated only in few pilot or retrospective studies. ${ }^{80-82}$ A significant suppression of the endogenous vasoconstrictor systems and a decrease of creatinine levels were recorded after TIPS in most patients with type 1 HRS. The rate of the creatinine decrease was slower than that usually obtained with albumin and vasoconstrictors. The reversal of type 1 HRS was observed in 57\%-71\% of patients. The recurrence of HRS was rare, provided that there was no TIPS dysfunction. The survival rates at 1 and 
3 months were within $50 \%-71 \%$ and $28 \%-64 \%$, respectively. More recently in a retrospective clinical study, it has been shown that in patients with type 1 HRS who were treated with TIPS had a longer survival than those who were treated without TIPS (vasoconstrictors and albumin or albumin alone and/or renal support). However, this result was affected by a relevant bias since patients who received TIPS has a lower baseline Child-Turcotte-Pugh score than non-TIPS patients. ${ }^{82}$

The prevalence of de novo hepatic encephalopathy or deterioration of previous hepatic encephalopathy after the insertion of TIPS ranges between $35 \%{ }^{81}$ and $71 \%{ }^{80}$ In addition, within 4 weeks after TIPS, patients experienced an average mild and transient deterioration of liver function tests ${ }^{81}$ particularly of the serum level of total bilirubin (from 3.1 to $4.4 \mathrm{mg} / \mathrm{dL}$ ). These observations raise the most important question in clinical practice of when TIPS should be used in patients with type 1-HRS? In all the studies, which were cited above, patients with history of severe encephalopathy or Child-Turcotte-Pugh score $>12$ were excluded. Moreover, if we take into account some of the more recent studies which compared TIPS and paracentesis in the treatment of refractory ascites, ${ }^{83-87}$ further contraindications to the use of TIPS were introduced including the following: a serum level of total bilirubin $>3$ or $5 \mathrm{mg} / \mathrm{dL}$, age $<5$ years, and the presence of cardiac failure or portopulmonary hypertension or hepatocellular carcinoma. Therefore, it seems that the applicability of TIPS is strongly limited in patients with type 1 HRS considering that it often occurs in the setting of acute on chronic liver failure. To confirm this, a controlled clinical trial on the use of TIPS as first-line treatment in patients with type 1 HRS is still lacking.

The results of vasoconstrictors and albumin in the treatment of type 1 HRS appear to be a quite an encouraging development for an effective treatment of type 1 HRS. Nevertheless, despite the normalization of serum creatinine as a result of this therapeutic option, GFR is still reduced in most responders to treatment. ${ }^{68}$ The effects of TIPS in patients with type 1 HRS treated initially with midodrine plus albumin have been evaluated in only 1 small pilot study. In 5 of 10 patients who achieved the reversal type 1 HRS by means of vasoconstrictors and albumin, the insertion of TIPS led to a complete normalization of GFR, as measured by inulin clearance $(96 \pm 20 \mathrm{~mL} / \mathrm{min})$. Furthermore, all these patients were alive after 6 months. ${ }^{69}$ Despite these promising results, a controlled clinical study on the use of TIPS as a second-line treatment in patients with type 1 HRS who respond, and in overall patients including those who do not respond, to vasoconstrictors and albumin is still lacking.

RRT, overall intermittent HD, and to a lesser extent continuous RRT (CRRT) such as continuous arteriovenous or venous-venous hemofiltration are used in the management of patients with type $1 \mathrm{HRS}$, especially in patients who failed vasoconstrictors and albumin and are candidates for OLT. Although there is no standard of practice when it comes to starting RRT in patients with cirrhosis and type $1 \mathrm{HRS}$, the decision to start is usually related to the failure of medical treatment to control volume overload, hyperklemia, metabolic acidosis or to the appearance of complications of uremia. Predictors of mortality after the starting of RRT have been found to be thrombocytopenia, hepatic encephalopathy, and the presence of comorbidities including sepsis, respiratory failure, and malignancy. There is still much controversy on the best modality of RRT. CRRT is claimed (1) to be better tolerated than HD in patients with liver failure as demonstrated by a better cardiovascular stability and (2) to have the advantage of removing proinflammatory cytokines including tumor necrosis factor $\alpha$, which have been implicated in the pathogenesis of HRS as well as in the exacerbation of liver damage. Nonetheless, the use of CRRT was associated with increased mortality in patients with HRS or with AKI including HRS. It should, however, be stressed that in both studies patients who received CRRT had always a greater severity of illness. ${ }^{88,89}$

However, to date, it is not possible to define the specific role of these techniques in the management of HRS since the evidence is still insufficient. The introduction of innovative techniques for extracorporeal liver function support like molecular adsorbent recirculating system (MARS) has made it possible to recover to some extent hepatic function, albeit transiently. In addition, when MARS was applied to the treatment of type 1 HRS, the ability of the procedure to remove vasoactive substances carried by albumin such as nitric oxide, tumor necrosis factor, and other proinflammatory cytokines and to reduce serum urea and serum creatinine were essential for its positive effects on systemic hemodynamics and renal function. From a clinical standpoint, such improvements have also produced a positive effect on 30-day survival in these patients $(37.5 \%$ vs $0 \%) .{ }^{90}$ Thereafter, a small noncontrolled clinical trial has shown that MARS was not effective in patients with type 1 HRS who had not responded before to vasoconstrictors and albumin. ${ }^{91}$ 


\section{Other therapeutic options for type 2 HRS}

On the clinical ground, patients with type 2 HRS do not present an acute renal failure, but rather refractory ascites. Therapeutic paracentesis is the first-choice treatment for refractory ascites, but TIPS is widely applied as an alternative to paracentesis in these patients whether type 2 HRS is detected or not. Up to now, 5 controlled studies have been published to this end, in which therapeutic paracentesis is compared with the use of TIPS. ${ }^{83-87}$ Overall these studies have demonstrated that (1) TIPS guarantees a better control of ascites, (2) the prevalence of encephalopathy is greater in patients who are treated with TIPS, (3) TIPS prevents the development of HRS, and (4) the impact on survival between the 2 treatments is not significantly different. Nevertheless, recent meta-analyses of available data show that TIPS significantly improve transplant-free survival in cirrhotic patients with refractory ascites. ${ }^{92-94}$ The effect of TIPS in patients with type 2 HRS has been specifically investigated in only 3 studies. ${ }^{80,81,95}$ In 2 retrospective studies from the same clinical center, a significant reduction of serum creatinine as well as an improvement in the control of ascites was observed. In addition, the 1-year survival was better than in those who did not receive any effective treatment ${ }^{79}$ and, even, in those who received other new therapeutic options but without a provided schedule (Figure 5). ${ }^{80}$

The effects of the vasoconstrictors and albumin in type 2 HRS treatment have not been extensively investigated. The percentage of response to treatment in terms of recovered renal function does not seem, however, to be different from that observed in patients with type $1 \mathrm{HRS},{ }^{63,64,96}$ whereas survival appears longer (100\% at 3 months).

\section{Prevention of HRS}

The most common precipitating factor of HRS is bacterial infection and, in particular, SBP. As a result of the development of better and quicker diagnostic strategies and more effective and safe antibiotic therapy over the last 20 years, SBP prognosis has greatly improved. Hospital mortality

- Be sure of the diagnosis by excluding the other forms of renal failure

- Look carefully for a precipitating event especially bacterial infections

- Remember that the main clinical problem is refractory ascites

- Adopt therapeutic paracentesis with plasma volume expansion by

means of albumin infusion as first choice treatment of ascites

- Use diuretics in order to reduce the frequency of repeated paracentesis only if they are tolerated

- Consider TIPS, when not contraindicated, if paracentesis becomes too frequent or ineffective

- Refer suitable patients to a liver transplant center

Figure 5 Key points in the management of type 2 hepatorenal syndrome. rates relating to this complication have in fact decreased from $50 \%$ to $20 \%$. Nevertheless, SBP-induced renal failure is still a common cause of death in these patients. ${ }^{11-13}$ The deterioration in renal function due to bacterial infection is thought to be the expression of a further reduction of effective circulating volume and in most cases it has the features of type 1 HRS. ${ }^{12}$ Therefore, the prevention of SBP in cirrhotic patients who are at high risk of developing either the bacterial infection or HRS is extremely important. Primary prophylaxis of SBP using long-term oral norfloxacin has been proven to be associated with a significant decrease in 1-year probability of development of SBP and type 1 HRS and a significant increase in 3-month and 1-year survival. ${ }^{97}$ In addition, when SBP occurs it has been observed that an association between effective antibiotic therapy and plasma volume expansion with albumin is capable of reducing the prevalence of SBP-induced renal failure and related mortality. ${ }^{98}$ Albumin appears able to prevent a further reduction of the effective circulating volume induced by the bacterial infection both by increasing the cardiac preload ${ }^{99}$ or by improving the cardiac contractility. ${ }^{100-102}$ This latter effect of albumin is probably related to its ability to bind $\mathrm{NO}^{100,101}$ and/or to inhibit the release of $\mathrm{NO}^{102}$ as a consequence of the infection which may further impair arterial vasodilation ${ }^{101}$ as well as develop a negative inotropic effect at the cardiac level. ${ }^{102}$ Another possible precipitating factor of HRS is acute alcoholic hepatitis. Treatment with pentoxifylline improves short-term survival in patients with severe alcoholic hepatitis. The benefit appears to be related to a significant decrease in the risk of developing HRS. ${ }^{103}$

Other effective methods for preventing of HRS are correct management of diuretic therapy and an adequate plasma volume expansion following therapeutic paracentesis in patients with cirrhosis and ascites (Table 1).

\section{Conclusions}

The use of vasoconstrictors and albumin represents a landmark in the treatment of HRS. Its development is closely related to the progressive focusing of the pathophysiology of HRS as a result of several experimental and clinical studies. It is really remarkable to observe that renal function may be recovered in patients with HRS by correcting the impairment of their cardiovascular function, namely the marked arterial splanchnic vasodilatation and the reduced cardiac output. The use of vasoconstrictors and albumin has also increased the survival in responders making it possible to increase the number of patients who undergo OLT. In addition, it has changed the outcome of OLT in these patients since it has 
Table I General recommendations for the rational medical therapy of ascites in patients with cirrhosis

I. Patients who develop moderate ascites for the first time should receive an aldosterone antagonist such as spironolactone alone, starting from $100 \mathrm{mg} /$ day increasing to maximum of $400 \mathrm{mg} /$ day (level I). In patients who do not respond to aldosterone antagonists, as defined by a reduction of body weight of less than $2 \mathrm{~kg} / \mathrm{wk}$, or in patients who develop hyperkalemia, furosemide should be added at an increasing dose from $40 \mathrm{mg} / \mathrm{day}$ to a maximum of $160 \mathrm{mg} /$ day (level I). Patients should undergo regular clinical and biochemical monitoring during the first month of treatment.

2. Patients who develop recurrent moderate ascites should be treated with a combination of an aldosterone antagonist plus furosemide, whose dose should be increased sequentially as above.

3. The maximum recommended weight loss during diuretic therapy should be $<0.5 \mathrm{~kg} /$ day in patients without edema, and $<\mathrm{l} \mathrm{kg} / \mathrm{day}$ in patients with significant edema.

4. The goal of long-term treatment is to keep patients free of ascites with the minimum dose of diuretics. Therefore, once the ascites has been largely resolved the dose of diuretics should be reduced or discontinued if possible.

5. Patients who are not responsive to top diuretic doses or those who develop serious complications under diuretic treatment should be checked for refractory ascites. First of all, the compliance with low-sodium diet should be checked by determining urine sodium excretion in these patients.

6. Since therapeutic paracentesis results in a more rapid resolution of ascites with a lower incidence of complications compared with diuretic treatment, paracentesis is the first-line therapy in patients with tense ascites.

7. Therapeutic paracentesis is also the first-line treatment in patients with refractory ascites.

8. Therapeutic paracentesis should be completed in a single session.

9. Since the removal of a large amount of ascitic fluid can cause impairment of circulatory function leading to renal failure and/or hyponatremia, it is necessary to prevent these circulatory changes. The best method to prevent circulatory dysfunction is the administration of albumin at a dose of $8 \mathrm{~g} / \mathrm{L}$ of ascitic fluid removed.

10. In patients undergoing therapeutic paracentesis of $>5 \mathrm{~L}$ of ascites, the use of alternative plasma expanders is not recommended since they are less effective in preventing postparacentesis circulatory dysfunction.

II. After therapeutic paracentesis, patients should receive the minimum dose of diuretics necessary to prevent the reaccumulation of ascites (level I).

reduced the post-OLT need of RRT and increased the postOLT survival. These results appear to be quite an encouraging development for an effective treatment of type 1 HRS. Nevertheless, it is important to recognize that a recovery of renal function can be achieved in less than $50 \%$ of patients with type $1 \mathrm{HRS}$ and, that the recovery of renal function may be partial also in patients who are defined as full responders on the basis of the normalization of the serum creatinine level and, overall, that the global effect on survival is slight. Therefore, we need to find out other therapeutic options, be they pharmacological or nonpharmacological to answer the most dramatic question we have today which is what we should do in patients with type 1 HRS who do not respond to vasoconstrictors and albumin.

As far as the nonpharmacological options are concerned, we can answer this question simply as follows. A high CTP score as well as a serum bilirubin $>10 \mathrm{mg} / \mathrm{dL}$, which has been identified as predictors of nonresponse to terlipressin and albumin, ${ }^{75,79}$ are, at the same time, contraindications to TIPS. As a consequence, RRT appears as the only rescue treatment in patients with type $1 \mathrm{HRS}$ who do not respond to vasoconstrictors and albumin. But, these contraindications are drawn from the trials comparing TIPS with paracentesis in the treatment of HRS. Here we are speaking about patients who will die within a few weeks without a rescue treatment. The lack of data on TIPS as well as on RRT in these patients still represents a relevant gap in current knowledge, particularly when patients who have developed type 1 HRS still are in the waiting list for OLT or when they still have a preserved performance status. To determine which of the two, TIPS or RRT, is better, it is important to consider not only survival but also quality of life as primary aim. For those who are candidates for OLT, the prioritization in the waiting list is on the basis of the MELD score. It is also noteworthy to observe that these 2 options are not mutually exclusive and that our choice between them in patients with type 1 HRS who do not respond to vasoconstrictors and albumin could vary according to the evolution of AKI. TIPS could be preferred in patient evaluated within 4 weeks from the time of onset of type 1 HRS and, thus, with high probability to recover renal function by reducing portal hypertension and improving systemic hemodynamics. On the contrary, RRT could be preferred in a patient evaluated after 4 weeks from the onset of type $1 \mathrm{HRS}$ when the evolution from a potentially reversible phenotype of AKI (type 1 HRS) towards a less reversible phenotype of AKI (ATN) is probably close to being completed. When the answer is RRT, other unanswered questions will follow such as (1) what modality of RRT we should choose among intermittent dialysis or CRRT? (2) what frequency and dose of RRT we should use? and (3) what anticoagulation protocol we should apply during RRT?

Finally, we need to decide what to do about patients who are not candidates for OLT. We need to assess whether the decision to initiate RRT in these patients could be considered 
futile, and whether we should give any recommendation on it or simply decide on a case-by-case basis.

We have no data that can support our decision in the current clinical practice. In fact, no data on the potential impact of these different therapeutic options on survival or on the MELD score are yet available. It does go without saying that these unanswered questions represent priorities for future research in this field.

\section{Disclosure}

The authors report no conflicts of interest in this work.

\section{References}

1. Gines A, Escorsell A, Gines P, et al. Incidence, predictive factors and prognosis of the hepatorenal syndrome in cirrhosis with ascites. Gastroenterology. 1993;105:229-236.

2. Schrier RW, Arroyo V, Bernardi M, Epstein M, Henriksen JH, Rodés J. Peripheral arterial vasodilation hypothesis: a proposal for the initiation of renal sodium and water retention in cirrhosis. Hepatology. 1988;8: $1151-1157$.

3. Kanel GC, Peters RL. Glomerular tubular reflux - a morphologic renal lesion associated with the hepatorenal syndrome. Hepatology. 1984;4: 242-246.

4. Arroyo V, Gines P, Gerbes AL, et al. Definition and diagnostic criteria of refractory ascites and hepatorenal syndrome in cirrhosis. Hepatology. 1996;23:164-176.

5. Salerno F, Gerbes A, Gines P, Wong F, Arroyo V. Diagnosis, prevention and treatment of the hepatorenal syndrome in cirrhosis. A consensus workshop of the International Ascites Club. Gut. 2007 (on line).

6. Praught ML, Shlipak MG. Are small changes in serum creatinine an important risk factor? Curr Opin Nephrol Hypertens. 2005;14: 265-270.

7. Levy MM, Macias WL, Vincent JL, et al. Early changes in organ function predict eventual survival in severe sepsis. Crit Care Med. 2005;33: 2194-2201.

8. Garcia-Tsao G, Parikh CR, Viola A. Acute kidney injury in cirrhosis. Hepatology. 2008;48:2064-2077.

9. Caregaro L, Menon F, Angeli P, et al. Limitations of serum creatinine level and creatinine clearance as filtration markers in cirrhosis. Arch Intern Med. 1994;154:201-205.

10. Gines P, Schier RW. Renal failure in cirrhosis. N Engl J Med. 2009;361: 1279-1290.

11. Follo A, Llovet JM, Navasa M, et al. Renal impairment after spontaneous bacterial peritonitis in cirrhosis: incidence, clinical course, predictive factors and prognosis. Hepatology. 1994;20:1495-1501.

12. Angeli P, Guarda S, Fasolato S, et al. Switch therapy with ciprofloxacin versus intravenous ceftazidime in the treatment of spontaneous bacterial peritonitis in patients with cirrhosis: similar efficacy at lower cost. Aliment Pharmacol Ther. 2006;23:75-84.

13. Fasolato S, Angeli P, Dallagnese L, et al. Renal failure and bacterial infections in patients with cirrhosis: epidemiology and clinical features. Hepatology. 2007;45:223-229.

14. Terra C, Guevara M, Torre A, et al. Renal failure in patients with cirrhosis and sepsis unrelated to spontaneous bacterial peritonitis: value of MELD score. Gastroenterology. 2005;129:1944-1953.

15. Maroto A, Ginès A, Saló J, et al. Diagnosis of functional kidney failure of cirrhosis with Doppler sonography: prognostic value of resistive index. Hepatology. 1994;20(4 Pt 1):839-844.

16. Wiest R, Groszmann RJ. The paradox of nitric oxide in cirrhosis and portal hypertension: too much, not enough. Hepatology. 2002;35:478-491.
17. Bolognesi M, Sacerdoti D, Piva A, et al. Carbon monoxide-mediated activation of large conductance calcium-activated potassium channels contributes to mesenteric vasodilation in cirrhotic rats. $J$ Pharmacol Exp Ther. 2007;321:187-194.

18. Sieber CC, Mosca PG, Groszmann RJ. Effect of somatostatin on mesenteric vascular resistance in normal and portal hypertensive rats. $\mathrm{Am}$ J Physiol. 1992;262:G274-G277.

19. Fernandez M, Garcia-Pagan JC, Casadevall M. Acute and chronic cyclooxygenase blockade in portal-hypertensive rats: influence on nitric oxide biosynthesis. Gastroenterology. 1996;110:1529-1535.

20. Guevara M, Gines P, Jimenez W, et al. Increased adrenomedullin levels in cirrhosis: relationship with hemodynamic abnormalities and vasoconstrictor systems. Gastroenterology. 1998;114:336-343.

21. Ros J, Claria J, To-Figueras J, et al. Endogenous cannabinoids: a new system involved in the homeostasis of arterial pressure in experimental cirrhosis in the rat. Gastroenterology. 2002;122:85-93.

22. Angeli P, Fernandez-Varo G, Dalla Libera V, et al. The role of nitric oxide in the pathogenesis of systemic and splanchnic vasodilation in cirrhotic rats before and after the onset of ascites. Liver. 2005;29: 429-437.

23. Arroyo V, Planas R, Gaya J, et al. Sympathetic nervous activity, reninangiotensin system and renal excretion of prostaglandin $\mathrm{E} 2$ in cirrhosis. Relationship to functional renal failure and sodium and water excretion. Eur J Clin Invest. 1983;13:271-278.

24. Henriksen JH, Ring-Larsen H. Hepatorenal disorders: role of the sympathetic nervous system. Semin Liver Dis. 1994;14:35-43.

25. Gentilini P, Romanelli RG, La Villa G, et al. Effects of low-dose captopril on renal hemodynamics and function in patients with cirrhosis of the liver. Gastroenterology. 1993;104:588-594.

26. Solis-Herruzo JA, Duran A, Favela V, et al. Effects of lumbar sympathetic block on kidney function in cirrhotic patients with hepatorenal syndrome. J Hepatol. 1987;5:167-173.

27. Piscaglia F, Zironi G, Gaiani S, et al. Relationship between splanchnic, peripheral and cardiac haemodynamics in liver cirrhosis of different degrees of severity. Eur J Gastroenterol Hepatol. 1997;9:799-804.

28. Fernandez-Seara J, Prieto J, Quiroga J, et al. Systemic and regional hemodynamics in patients with liver cirrhosis and ascites with and without functional renal failure. Gastroenterology. 1989;97:1304-1312.

29. Angeli P, Volpin R, Piovan D, et al. Acute effects of an oral administration of midodrine an $\alpha$-adrenergic agonist, on renal hemodynamics and renal function in cirrhotic patients with ascites. Hepatology. 1998;28: 937-943.

30. Tristani FE, Cohn JN. Systemic and renal hemodynamics in oliguric hepatic failure: effect of volume expansion. J Clin Invest. 1967;46: 1894-1906.

31. Lebrec D, Kotelanski B, Cohn JN. Splanchnic hemodynamic factors in cirrhosis with refractory ascites. J Lab Clin Med. 1979;93:301-309.

32. Colle I, Moreau R, Pessione F. Relatioships between haemodynamic alterations and the development of ascites or refractory ascites in patients with cirrhosis. Eur J Gastroenterol Hepatol. 2001;13: 251-256.

33. Ruiz del Arbol L, Urman J, Fernandez J, et al. Systemic, renal and hepatic haemodynamic derangement in cirrhotic patients with spontaneous bacterial peritonitis. Hepatology. 2003;38:1210-1218.

34. Krag A, Bendtsen F, Henriksen JH, Moller S. Low cardiac output predicts development of hepatorenal syndrome and survival in patients with cirrhosis and ascites. Gut. 2010;59:105-110.

35. Tsai MH, Peng YS, Chen YC, et al. Adrenal insufficiency in patients with cirrhosis, severe sepsis and septic shock. Hepatology. 2006;43: 673-681.

36. Fernandez J, Escorsell A, Zabalza M, et al. Adrenal insufficiency in patients with cirrhosis and septic shock: effect of treatment with hydrocortisone on survival. Hepatology. 2006;44:1288-1295.

37. Ma ZH, Lee SS. Cirrhotic cardiomyopathy: getting to the heart of the matter. Hepatology. 1996;24:451-459. 
38. Jalan R, Forrest EH, Redhead DN, Dillon JF, Hayes PC. Reduction in renal blood flow following acute increase in the portal pressure: evidence for the existence of a hepatorenal reflex in man? Gut. 1997;40:664-670.

39. Soper CPR, Latif AB, Bendino MR. Amelioration of hepatorenal syndrome with selective endothelin-A antagonist. Lancet. 1996;347:1842-1843.

40. Wong F, Moore K, Dingemanse J, Jalan R. Lack of renal improvement with non selective endothelin antagonism with tezosentan in type 2 hepatorenal syndrome. Hepatology. 2008;47:160-168.

41. Rimola A, Gines P, Arroyo V, et al. Urinary excretion of 6-keto-prostaglandin $\mathrm{F} 1 \alpha$, thromboxane B2 and prostaglandin E2 in cirrhosis with ascites. J Hepatol. 1986;3:111-117.

42. Zipster RD, Radvan GH, Kronborg IJ, et al. Urinary thromboxane B2 and prostaglandin E2 in the hepatorenal syndrome: evidence for increased vasoconstrictor and decreased vasodilator factors. Gastroenterology. 1983;84:697-703

43. Moore KP, Taylor GW, Maltby NH, et al. Increased production of cysteinyl leukotrienes in hepatorenal syndrome. J Hepatol. 1990;11: 263-271.

44. Sacerdoti D, Balazy M, Angeli P, et al. Eicosanoid excretion in hepatic cirrhosis. Predominance of 20-HETE. J Clin Invest. 1997;100: 1264-1270.

45. Ros J, Claria J, Jimenez W, et al. Role of nitric oxide and prostacyclin in the control of renal perfusion in experimental cirrhosis. Hepatology. 1995;22: 915-920.

46. Angeli P, Jimenez W, Arroyo V, et al. Renal effects of endogenous natriuretic peptides receptors blockade in cirrhotic rats with ascites. Hepatology. 1994;20:948-954.

47. Laffi G, Daskalopoulos G, Kronborg I, et al. Effects of sulindac and ibuprofen in patients with cirrhosis and ascites. An explanation for the renal-sparing effect of sulindac. Gastroenterology. 1986;90: $182-187$.

48. Gonwa TA, Klintmalm GB, Levy M, et al. Impact of pre-transplant renal function on survival after liver transplantation. Transplantation. 1995;59: 361-365.

49. Navasa M, Feu F, Garcia-Pagan JC, et al. Hemodynamic and humoral changes after liver-transplantation in patients with cirrhosis. Hepatology. $1993 ; 17: 355-360$.

50. Schlitt HJ, Barkmann A, Boker KH, et al. Replacement of calcineurin inhibitors with mycophenolate mofetil in liver-transplant patients with renal dysfunction: a randomised controlled study. Lancet. 2001;357: 587-591.

51. Restuccia T, Ortega R, Guevara M, et al. Effects of treatment of hepatorenal syndrome before transplantation on post-transplantation outcome. A case control study. Liver Transpl. 2004;40:140-146.

52. Davis CL, Feng S, Sung R, et al. Simultaneous liver-kidney transplantation: evaluation to decision making. Am J Transplant. 2007;7: 1702-1709.

53. Eason JD, Gonwa TA, Davies CL, et al. Proceedings of consensus conference on simultaneous liver kidney ransplantation (SLK). Am J Transplant. 2008;8:2243-2251.

54. Xu X, Ling Q, Zhang M, et al. Outcome of patients with hepatorenal syndrome type 1 after liver transplantation: Hangzhou experience. Transplantation. 2009;87:1514-1519.

55. Marik PE, Wood K, Starzl TE. The course of type 1 hepato-renal syndrome post liver transplantation. Nephrol Dial Transplant. 2006;21: 478-482.

56. Guevara M, Gines P, Fernandez-Esparrach G, et al. Reversibility of hepatorenal syndrome by prolonged administration of ornipressin and plasma volume expansion. Hepatology. 1998;27:35-41.

57. Gulberg V, Bilzer M, Gerbes AL. Long-term therapy and retreatment of hepatorenal syndrome type 1 with ornipressin and albumin. Hepatology. 1999;30:870-875.

58. Ganne-Carrie N, Hadegue A, Mathurin P, et al. Hepatorenal syndrome: long-term treatment with terlipressin as bridge to liver transplantation. Dig Dis Sci. 1996;41:1054-1056.
59. Le Moine O, El Nawar A, Jagodzinski R, et al. Treatment with terlipressin as a bridge to transplantation in a patient with hepatorenal syndrome. Acta Gastroenterol Belg. 1998;61:268-270.

60. Duhamel C, Mauillon J, Berkelmans J, Bourienne A, Tranvuez JL. Hepatorenal syndrome in cirrhotic patients: terlipressin is a safe and efficient treatment; propranolol and digitalic treatment: precipitating and preventing factors? Am J Gastroenterol. 2000;95:2984-2985.

61. Halimi C, Bonnard P, Bernard B, et al. Effect of terlipressin (glypressin) on hepatorenal syndrome in cirrhotic patients: results of a multicenter pilot study. Eur J Gastroenterol Hepatol. 2002;14:153-158.

62. Mulkay JP, Louis H, Donckier V, et al. Long-term terlipressin administration improves renal function in cirrhotic patients with type 1 hepatorenal syndrome: a pilot study. Acta Gastroelerologica Belg. 2001;64: $15-19$.

63. Uriz J, Gines P, Cardenas A, et al. Terlipressin plus albumin infusion: an effective and safe therapy of hepatorenal syndrome. J Hepatol. 2000;33: 43-48.

64. Ortega R, Giniss P, Uriz J, et al. Terlipressin therapy with and without albumin for patients with hepatorenal syndrome: results of a prospective, nonrandomized study. Hepatology. 2002;36:941-948.

65. Solanki P, Chawala A, Garg R, et al. Beneficial effects of terlipressin in hepatorenal syndrome: a prospective, randomized placebo-controlled clinical trial. J Gastroenterol Hepatol. 2003;18:152-156.

66. Moreau R, Durand F, Poynard T, et al. Terlipressin in patients with cirrhosis and type 1 hepatorenal syndrome: a retrospective multicenter study. Gastroenterology. 2002;122:923-930.

67. Colle I, Durand F, Pessione F, et al. Clinical course, predictive factors and prognosis in patients with cirrhosis and type 1 hepatorenal syndrome treated with terlipressin: a retrospective analysis. J Gastroenterol Hepatol. 2002;17:882-888.

68. Angeli P, Volpin R, Gerunda G, et al. Reversal of type 1 hepatorenal syndrome (HRS) with the combined administration of midodrine and octreotide. Hepatology. 1999;29:1690-1697.

69. Wong F, Pantea L, Sniderman K. Midodrine, octreotide, albumin, and TIPS in selected patients with cirrhosis and type 1 hepatorenal syndrome. Hepatology. 2004;40:55-64.

70. Esrailian E, Pantageo ER, Kyulo NL, Hu KQ, Runyon BA. Octretide/ midodrine therapy significantly improves renal function and 30-day survival in patients with type 1 hepatorenal syndrome. Dig Dis Sci. 2007;52: $742-748$.

71. Skagen C, Einstein M, Lucey MR, Said A. Combination treatment with ocreotide, midodrine, and albumin improves survival in patients with type 1 and type 2 hepatorenal syndrome. J Clin Gastroenterol. 2009;43: 680-685.

72. Devoux C, Zanditenas D, Hezode C, et al. Effects of noradrenalin and albumin in patients with type 1 hepatorenal syndrome: a pilot study. Hepatology. 2002;36:374-380.

73. Sharma P, Kumar A, Sharma BC, Sarin SK. An open label, pilot, randomized controlled trial of noradrenaline versus terlipressin in the treatment of type 1 hepatorenal syndrome and predictors of response. Am J Gastroenterol. 2008;103:1689-1697.

74. Mandal AK, Lansing M, Fahmy A. Acute tubular necrosis in hepatorenal syndrome: an electron microscopy study. Am J Kidney Dis. 1982;23: 363-374.

75. Sanyal A, Boyer T, Garcia-Tsao G, et al. A randomized, prospective, double blind placebo-controlled trial of terlipressin for type 1 hepatorenal syndrome. Gastroenterology. 2008;134:1360-1368.

76. Martin-Llahi MM, Pepin MN, Guevara M, et al. Terlipressin and albumin versus albumin in patients with cirrhosis and hepatorenal syndrome: a randomized study. Gastroenterology. 2008;134:1352-1359.

77. Gluud LL, Christensen K, Krag A. Systematic review of randomized trials on vasoconstrictor drugs for hepatorenal syndrome. 2010;51:576-584.

78. Angeli P. Review article: prognosis of hepatorenal syndrome - has it changed with current practice? Aliment Pharmacol Ther. 2004;20 Suppl 3: $1-4$. 
79. Nazar A, Pereira GH, Guevara M, et al. Predictors of response to therapy with terlipressin and albumin in patients with cirrhosis and type 1 hepatorenal syndrome. Hepatology. 2010;51:219-226.

80. Guevara M, Gines P, Bandi JC, et al. Transjugular intrahepatic portosystemic shunt in hepatorenal syndrome: effects on renal function and vasoactive systems. Hepatology. 1998;28:416-422.

81. Brensing KA, Textor J, Perz J, et al. Long term outcome after transjugular intrahepatic portosystemic stent-shunt in non-transplant cirrhotics with hepatorenal syndrome; a phase II study. Gut. 2000;47: 288-295.

82. Appenrodt B, Zielinski J, Bresing KA, Heller J, Sauerbruch T, Schepke M. Degree of hepatic dysfunction and improvement of renal function predict survival in patients with HRS type 1: a retrospective analysis. Eur J Gastroenterol Hepatol. 2009;21:1428-1432.

83. Lebrec D, Giuily N, Hadengue A, et al. Transjugular intrahepatic portosystemic shunts: comparison with paracentesis in patients with cirrhosis and refractory ascites. J Hepatol. 1996;25:135-144.

84. Rossle M, Ochs A, Gulberg V, et al. A comparison of paracentesis and transjugular intrahepatic portosystemic shunting in patients with ascites. N Eng J Med. 2000;342:1701-1707.

85. Gines P, Uriz J, Calahorra B, et al. Transjugular intrahepatic portosystemic shunting versus paracentesis plus albumin for refractory ascites in cirrhosis. Gastroenterology. 2002;123:1839-1847.

86. Sanyal AJ, Genning C, Reddy KR, et al. The North American Study for the treatment of refractory ascites. Gastroenterology. 2003;124: 634-641.

87. Salerno F, Merli M, Riggio O, et al. Randomized controlled study of TIPS versus paracentesis plus albumin in cirrhosis with severe ascites. Hepatology. 2004;40:629-635.

88. Witzke O, Baumann M, Patschan D, et al. Which patients benefit from haemodialysis therapy in hepatorenal syndrome? J Gastroenterol Hepatol. 2004;19:1369-1373.

89. Gonwa TA, Mai ML, Melton LB, et al. Renal replacement therapy and orthotopic liver transplantation. The role of continuous veno-venous hemodialysis. Transplantation. 2001;71:1424-1428.

90. Mitzner SR, Stange J, Klammt S, et al. Improvement of hepatorenal syndrome with extracorporeal albumin dialysis MARS: results of a prospective, randomized controlled clinical trial. Liver Transpl. 2000;6: 277-286.

91. Wong F, Raina N, Richardson R. Molecular adsorbent recirculating system is ineffective in the management of type 1 hepatorenal syndrome in patients with cirrhosis with ascites who have failed vasoconstrictor therapy. Gut. 2010;59:381-386.

92. Saab S, Nieto JM, Ly D, et al. TIPS versus paracentesis for cirrhotic patients with refractory ascites. Cochrane Database Syst Rev. 2004;3:CD004889.
93. D’Amico G, Luca A, Morabito A, et al. Uncovered transjugular intrahepatic portosystemic shunt for refractory ascites: a meta-analysis. Gastroenterology. 2005;129:1282-1293.

94. Salerno F, Cammà C, Enea M, Rossle M, Wong F. Transjugular intrahepatic portosystemic shunt for refractory ascites: a meta-analysis of individual patient data. Gastroenterology. 2007;133:825-834.

95. Testino G, Ferro C, Sumberaz A, et al. Type-2 hepatorenal syndrome and refractory ascites: role of transjugular intrahepatic portosystemic stent-shunt in eighteen patients with advanced cirrhosis awaiting orthotopic liver transplantation. Hepatogastroenterology. 2003;50: 1753-1755.

96. Alessandria C, Venon WD, Marzano A, Barletti C, Fadda M, Rizzetto M. Renal failure in cirrhotic patients: role of terlipressin in clinical approach to hepatorenal syndrome type 2. Eur J Gastroenterol Hepatol. 2002;14: 1363-1368.

97. Fernandez J, Navasa M, Planas R, et al. Primary prophylaxis of spontaneous bacterial peritonitis delays hepatorenal syndrome and improves survival in cirrhosis. Gastroenterology. 2007;133:818-824.

98. Sort P, Navasa M, Arroyo V, et al. Effect of intravenous albumin on renal impairment and mortality in patients with cirrhosis and spontaneous bacterial peritonitis. N Engl J Med. 1999;341:403-409.

99. Brinch K, Moller S, Bendtsen F, Becker U, Henriksen JH. Plasma volume expansion by albumin in cirrhosis. Relation to blood volume distribution, arterial compliance and severity of disease. J Hepatol. 2003;39: 24-31.

100. Evans TW. Review article: albumin as a drug-biological effects of albumin unrelated to oncotic pressure. Aliment Pharmacol Ther. 2002;16 Suppl 5:6-11.

101. Fernandez J, Navasa M, Garcia-Pagan JC, et al. Effect of intravenous albumin on systemic and hepatic hemodynamics and vasoactive neurohormonal systems in patients with cirrhosis and spontaneous bacterial peritonitis. J Hepatol. 2004;41:384-390.

102. Meziani F, Kremer H, Tesse A, et al. Human serum albumin improves arterial dysfunction during early resuscitation in mouse endotoxic model via reduced oxidative and nitrosative stresses. Am J Pathol. 2007; 171:1753-1761.

103. Akriviadis E, Botla R, Briggs W, Han S, Reynolds T, Shakil O. Pentoxifylline improves short-term survival in severe acute alcoholic hepatitis: a double-blind, placebo-controlled trial. Gastroenterology. 2000;119: 1637-1648.

104. Chertow GM, Burdick E, Honour M, Bonventre JV, Bates DW. Acute kidney injury, mortality, length of stay, and costs in hospitalized patients. J Am Soc Nephrol. 2005;16:3365-3370.
Hepatic Medicine: Evidence and Research

\section{Publish your work in this journal}

Hepatic Medicine: Evidence and Research is an international, peerreviewed, open access journal covering all aspects of adult and pediatric hepatology in the clinic and laboratory including the following topics: Pathology, pathophysiology of hepatic disease; Investigation and treatment of hepatic disease; Pharmacology of drugs used for

\section{Dovepress}

the treatment of hepatic disease. Issues of patient safety and quality of care will also be considered. The manuscript management system is completely online and includes a very quick and fair peer-review system, which is all easy to use. Visit http://www.dovepress.com/ testimonials.php to read real quotes from published authors. 